\title{
Alloy design as an inverse problem of cluster expansion models
}

\author{
Larsen, Peter Mahler; Kalidindi, Arvind R.; Schmidt, Søren; Schuh, Christopher A.
}

\section{Published in:}

Acta Materialia

Link to article, DOI:

10.1016/j.actamat.2017.08.008

Publication date:

2017

Document Version

Peer reviewed version

Link back to DTU Orbit

Citation (APA):

Larsen, P. M., Kalidindi, A. R., Schmidt, S., \& Schuh, C. A. (2017). Alloy design as an inverse problem of cluster expansion models. Acta Materialia, 139, 254-260. https://doi.org/10.1016/j.actamat.2017.08.008

\section{General rights}

Copyright and moral rights for the publications made accessible in the public portal are retained by the authors and/or other copyright owners and it is a condition of accessing publications that users recognise and abide by the legal requirements associated with these rights.

- Users may download and print one copy of any publication from the public portal for the purpose of private study or research.

- You may not further distribute the material or use it for any profit-making activity or commercial gain

- You may freely distribute the URL identifying the publication in the public portal

If you believe that this document breaches copyright please contact us providing details, and we will remove access to the work immediately and investigate your claim. 


\section{Accepted Manuscript}

Alloy design as an inverse problem of cluster expansion models

Peter Mahler Larsen, Arvind R. Kalidindi, Søren Schmidt, Christopher A. Schuh

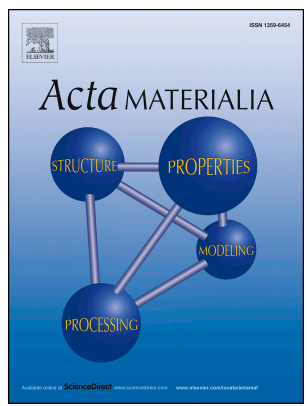

PII:

S1359-6454(17)30651-1

DOI:

10.1016/j.actamat.2017.08.008

Reference: $\quad$ AM 13969

To appear in: Acta Materialia

Received Date: 20 June 2017

Revised Date: 3 August 2017

Accepted Date: 4 August 2017

Please cite this article as: P.M. Larsen, A.R. Kalidindi, Sø. Schmidt, C.A. Schuh, Alloy design as an inverse problem of cluster expansion models, Acta Materialia (2017), doi: 10.1016/ j.actamat.2017.08.008.

This is a PDF file of an unedited manuscript that has been accepted for publication. As a service to our customers we are providing this early version of the manuscript. The manuscript will undergo copyediting, typesetting, and review of the resulting proof before it is published in its final form. Please note that during the production process errors may be discovered which could affect the content, and all legal disclaimers that apply to the journal pertain. 


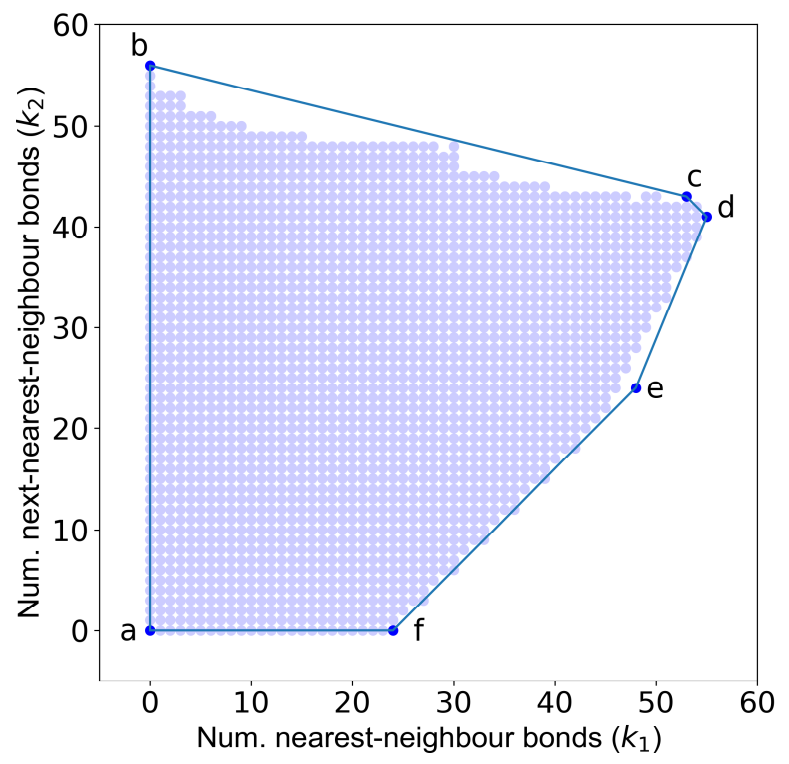

(a)

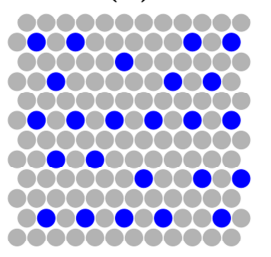

(d)

00000000000 00000000000

000900000 00000000 00000 000000 000000000 000000000000 (b)

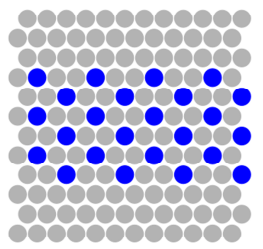

(e)

00000000000 000000000

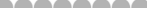
(2) $\$ 00000000$ 0000000000

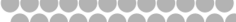
0000000000 (c)

00000000000 80000000000 00000000 000000 000000 000000000 00000000000 00000000000

(f)

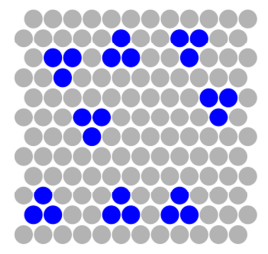
Num. nearest-neighbour bonds $\left(k_{1}\right)$ 


\title{
Alloy Design as an Inverse Problem of Cluster Expansion Models
}

\author{
Peter Mahler Larsen ${ }^{\mathrm{a}, \mathrm{b}}$, Arvind R. Kalidindi ${ }^{\mathrm{a}}$, Søren Schmidt ${ }^{\mathrm{b}}$, Christopher A. Schuh ${ }^{\mathrm{a},{ }^{*}}$ \\ ${ }^{a}$ Department of Materials Science and Engineering, Massachusetts Institute of Technology, Cambridge, MA \\ 02139, USA \\ ${ }^{b}$ Department of Physics, Technical University of Denmark, 2800 Kgs. Lyngby, Denmark \\ *Corresponding author
}

Email address: schuh@mit.edu (Christopher A. Schuh)

\begin{abstract}
Central to a lattice model of an alloy system is the description of the energy of a given atomic configuration, which can be conveniently developed through a cluster expansion. Given a specific cluster expansion, the ground state of the lattice model at $0 \mathrm{~K}$ can be solved by finding the configuration of solutes that minimizes the energy of the system. In this paper, we develop a method for solving the inverse lattice problem, where, given a broad class of potential, we find the ground states for all possible values of the effective cluster interaction energies. To do so, we formulate the inverse problem in terms of energetically distinct configurations, using a constraint satisfaction model to identify constructible configurations, and show that a convex hull can be used to identify ground states. To demonstrate the approach, we solve for all ground states for a binary alloy in a 2D hexagonal lattice both with and without an interface, based on pairwise interactions.
\end{abstract}

Keywords: Cluster expansion, Computational thermodynamics, Alloys, Microstructure design, Phase diagrams

\section{Introduction}

Statistical mechanics models of alloys assign probabilities to the possible configurations of alloying elements and, based on a Maxwell-Boltzmann distribution, determine the equilibrium state of the alloy. Describing the configuration space of the alloy inevitably requires some approximation. The simplest models rely on assumptions of random distributions of each alloying element, either in the entire system, as in an ideal solution model, or within any phase as in a regular solution model. A major improvement to capturing configurational degrees of freedom is a lattice model, also known as the generalized Ising model [1] or Cluster Expansion [2] (CE) model, where each lattice site represents a single atom of a particular element with a pseudo-spin occupancy variable $\sigma_{i}$ that, for the binary case, is -1 or +1 for a solute or solvent atom, respectively. The energy of an alloy configuration, $\boldsymbol{\sigma}$, in the lattice model with a cluster expansion interatomic potential, $\mathbf{V}$, can generally be defined by the Hamiltonian [3]:

$$
H \boldsymbol{\sigma}, \mathbf{V}=V_{0}+\sum_{c \in \mathbf{C}} V_{c} \boldsymbol{\sigma}_{c}
$$

where $\mathbf{C}$ is the set of clusters for which effective cluster interactions (ECIs), $V_{c}$, are prescribed. $\boldsymbol{\sigma}_{c}$ is a generic cluster function defined as the product of $\sigma_{i}$ over all sites in a cluster and captures the solute configuration of the cluster. While a fixed site geometry still constrains the configuration space considered, lattice models are a remarkably accurate tool for developing phase diagrams of alloys, where a cluster expansion can be performed from ab-initio calculations $[2,4,5,6,7,8,9,10,11,12$, 13].

The $0 \mathrm{~K}$ phase diagram is determined by the alloy configurations that minimize Equation (1) at different solute concentrations. The problem of merely solving for the minimum energy configurations we term the 'forward problem'. Conversely, the much more complex 'inverse problem' is the one underlying alloy design, where, given a desired alloy configuration the objective is to determine which alloy systems, if any, possess this configuration as an equilibrium state. The possible ground states of the alloy model are those for which there exists a parameter set $\mathbf{V}$ that minimizes energy according to Equation (1). While the relationship between energy and the cluster 
functions is linear, finding all possible ground states is difficult due to the constraints imposed on the cluster functions by the lattice, namely the integrality constraint of equalling -1 or 1 .

Allen and Cahn were able to solve this inverse problem for body-centered cubic and face-centered cubic lattices where the interatomic potential is constrained to nearest-neighbor and next-nearestneighbor pairwise bonds [14]. Their approach and the more general polytope method $[15,16,17,18]$ circumvent the integrality constraint by first selecting one base cluster (e.g. an octahedron for the body-centered cubic lattice), and then determining all possible ways in which the alloying elements can occupy sites in this cluster, assigning each configuration on the cluster a probability weight from 0 to 1 . Since probability is a continuous function, this problem can be solved by standard linear programming techniques to determine all possible equilibrium states.

However, not all combinations of probabilities for each cluster are constructible in a larger lattice, and thus additional rules must be defined to ensure that a ground state determined in this way represents a physical system. In a crystalline phase with short range interactions, these rules can be reasonably simple, but in more complex systems constructibility can be a cumbersome problem [18]. Inverse problems in certain disordered systems, such as hyperuniform glasses, have been studied to determine the interatomic potentials that lead to disordered ground states [19, 20], but are beyond the scope of a standard cluster expansion representation. There has been growing interest in studying chemical ordering at interfaces, such as grain boundaries, and constructing phase diagrams (also known as complexion diagrams) of the segregation and ordering at these interfaces [21, 22, 23, 24, 25, 26, 27, $28,29,30]$. Such problems have an inhomogeneous cluster expansion, since clusters at an interface have different energies than ones in the crystal, which makes use of the polytope method more challenging. Even in crystalline phases, considering clusters with longer range interactions can be challenging within the polytope method as a larger base cluster must be chosen leading to a larger number of potential configurations and more complex constructibility considerations.

The forward problem of finding ground states in complex systems has recently been formulated by Huang et al. [31] as a class of problem known as pseudo-Boolean optimization [32] (PBO). PBO models, which consist of an objective function to be optimized and a set of constraints to be satisfied, greatly improve the generality and speed of solving lattice models given a cluster expansion. In this paper, we present a framework for solving the inverse problem of the lattice model directly in the configuration space of the lattice using a constraint satisfaction model. We then investigate the ground state ordered states in a 2D hexagonal lattice both with and without an interface to demonstrate how all possible configurations with a given potential form can be calculated.

\section{The Inverse Problem in Lattice Models}

The forward problem asks: for a specified $\mathbf{V}$, what is the ground state configuration? A configuration $\boldsymbol{\sigma}$ is a ground state if it has a lower energy than all other configurations:

$$
H \boldsymbol{\sigma}, \mathbf{V}<H \quad \boldsymbol{\sigma}^{\prime}, \mathbf{V} \forall \boldsymbol{\sigma}^{\prime} \neq \boldsymbol{\sigma}
$$

Then, the inverse problem asks: for a specified configuration $\boldsymbol{\sigma}$, does there exist a set $\mathbf{V}$ for which $\boldsymbol{\sigma}$ is the ground state? Here, the space of interactions being considered (e.g. nearest neighbor, nextnearest neighbor, etc.) is constant. We denote such a configuration as minimizing. It must satisfy the condition:

$$
\exists \mathbf{V} \quad \text { s.t. } \quad H \quad \boldsymbol{\sigma}, \mathbf{V}<H \quad \boldsymbol{\sigma}^{\prime}, \mathbf{V} \quad \forall \boldsymbol{\sigma}^{\prime} \neq \boldsymbol{\sigma}
$$

Thus, to solve the inverse problem, all possible ECIs as well as all possible configurations must be considered in order to find the ground states. In order to make this tractable, we will first reduce the configuration space to a smaller, abstract space, and then use arguments based on principles of convexity to identify minimizing configurations.

In order to reduce the configuration space, we note that the Hamiltonian in Equation (1) can be expanded to lattice-gas [33] form: 


$$
H \mathbf{x}, \mathbf{E}=E_{0}+\sum_{j} \sum_{\mathbf{c} \in \mathbf{C}_{j}} \prod_{i \in \mathbf{c}} E_{j} x_{i}
$$

where spin variables $\sigma_{i} \in-1,1$ of the cluster function have been replaced by a binary variable $x_{i} \in 0,1$ using the relation $\sigma_{i}=2 x_{i}-1$, and $\mathbf{E}$ is the equivalent set of ECIs. For each cluster instance we can then define a binary variable $y_{\mathbf{c}}=\prod_{i \in \mathbf{c}} x_{i}$ which denotes cluster instance activity, where 0 and 1 denote an inactive and active cluster instance respectively. Using Equation (4), the Hamiltonian can then be rewritten in the number of active cluster instance (ACI) counts:

$$
H \mathbf{x}, \mathbf{E}=\langle\mathbf{E}, \mathbf{N}\rangle
$$

where $\mathbf{N}=\left\{\sum_{\mathbf{c} \in \mathbf{C}_{1}} y_{\mathbf{c}}, \sum_{\mathbf{c} \in \mathbf{C}_{2}} y_{\mathbf{c}}, \sum_{\mathbf{c} \in \mathbf{C}_{3}} y_{\mathbf{c}}, \ldots\right\}$ is the vector of ACI counts (ACI vector). Representing a configuration as an ACI vector is a much more compact description and permits a simpler expression of the Hamiltonian. Using this parametrization, we define the 'energy space' $V \mathbf{N}$ of a configuration as the region of ECI space for which it is a ground state:

$$
V(\mathbf{N})=\left\{\mathbf{E} \mid\langle\mathbf{E}, \mathbf{N}\rangle<\left\langle\mathbf{E}, \mathbf{N}^{\prime}\right\rangle \forall \mathbf{N}^{\prime} \neq \mathbf{N}\right\}
$$

where $\mathbf{N}$ and $\mathbf{N}^{\prime}$ denote constructible bond count vectors with the same number of solute atoms.

Determination of the minimizing configurations proceeds by identifying the convex hull [34] of configurations. The maximum principle [35] states that the maximum (or equally, minimum) of any convex function on a compact convex set is attained at the boundary of the set. Thus, in order to exploit the maximum principle, we require a convex function of a convex compact set. If we relax the implicit integrality constraints on $\mathbf{N}$, the inner product in Equation (5) is a sum of linear functions, which is a convex function. Next, let $\mathbf{S}=\mathbf{N}_{1}, \mathbf{N}_{2}, \mathbf{N}_{3}, \ldots$ be the set of all constructible ACI vectors for a given set of clusters. Then, the convex hull of $S$ is by definition a compact convex set. By restricting the domain ( $\mathbf{N}$ ) of the Hamiltonian in Equation (5) to the convex hull of $\mathbf{S}$, we satisfy the necessary conditions of the maximum principle. As such, we can state that all possible ground states for a given $\mathbf{E}$ must lie on the boundary of the convex hull of $\mathbf{S}$. For practical reasons we can tighten the definition: any minimizing configuration must lie on a vertex of the convex hull of $\mathbf{S}$. We can do this since any configuration which lies on a plane of the convex hull has a zero energy space according to Equation (6).

Figure 1: Different configurations with the same stoichiometry in a periodic 1D lattice. Bonds exist between adjacent sites, which are occupied by either A-type (gray) or B-type (blue) atoms. The configurations shown here are energetically unique configurations for 12 A-type and 8 B-type atoms. Of the eight configurations, only two, (a) and (h), are possible ground state configurations, as they have extreme values of $k_{1}$ (the number of nearest-neighbor B-B bonds).

What the above means is that solving the inverse problem amounts to finding all states that lie on the vertices of the convex hull of cluster space, which is a space where each axis is the number of counts of a given cluster. We illustrate this concept with a 1D example. Figure 1 shows a periodic lattice in $\mathbb{R}^{1}$, with bonds between adjacent sites only, and whose sites are occupied by either A-type or B-type atoms. Given this energetic model, there are two types of clusters: 1-body and nearest-neighbor 2body clusters. For a fixed concentration of B-type atoms, though, the contribution of 1-body clusters is the same in each case, meaning there is only one linearly independent cluster. The ACI vector of any configuration in this model can therefore be written as a $1 \mathrm{D}$ vector, $\mathbf{N}=k_{1}$ where $k_{1}$ is the number of nearest-neighbor B-B bonds. As such, the convex hull of all constructible ACI vectors 
consists of two points. These states, which constitute the minimizing configurations of the lattice, correspond to preferences for heteroatomic bonds (Figure 1a) and for homoatomic bonds (Figure 1h).

\section{Enumerating Constructible Configurations}

In order to determine the convex hull of cluster space and find the set of minimizing configurations, we need to consider all possible ACI vectors. A naive method is to simply construct all possible configurations at a specified number of solute atoms, from which the set of ACI vectors can be obtained. This method, however, leads to many redundant calculations. Consider the 1D example in Section 2; with $n_{\mathrm{s}}$ lattice sites and $n_{\mathrm{b}}$ B-type atoms there are $\left(\begin{array}{l}n_{\mathrm{s}} \\ n_{\mathrm{b}}\end{array}\right)$ unique configurations, yet only $n_{\mathrm{b}}$ unique ACI vectors. For the illustrated example with $n_{\mathrm{s}}=20$ and $n_{\mathrm{b}}=8$ there are 125970 configurations of which only 8 have unique ACI vectors.

Instead, the ACI vectors can be enumerated directly, which amounts to considering only energetically distinct configurations. This approach poses a new challenge: not all ACI vectors are constructible. For example, for $n_{\mathrm{b}}<n_{\mathrm{s}}$ all ACIs with $k_{1} \geq n_{\mathrm{b}}$ are inconstructible. In the $1 \mathrm{D}$ example this constraint is obvious, but in higher dimensional lattices or lattices with different site types the conditions for constructibility are non-trivial.

\begin{tabular}{|l|c|r|l|r|}
\hline Variables: & $x_{i} \in 0,1$ & $\forall i$ & Type of atom site $i$ & $(7)$ \\
\hline & $y_{\mathbf{c}} \in 0,1$ & $\forall \mathbf{c}$ & Cluster instance variable & $(8)$ \\
\hline Parameters: & $\mathbf{N} \in \mathbb{N}_{0}^{\mathbf{J} l}$ & & ACI vector & $(9)$ \\
\hline & $n_{\mathrm{b}} \in \mathbb{N}_{0}$ & & Number of B-type atoms & $(10)$ \\
\hline Constraints: & $\sum_{i} x_{i}=n_{\mathrm{b}}$ & & Fixed stoichiometry & $(11)$ \\
& $y_{\mathbf{c}}=\operatorname{AND}\left(\left\{x_{i} \mid i \in \mathbf{c}\right\}\right)$ & $\forall \mathbf{c}$ & Cluster instance activity & $(12)$ \\
\hline & $\sum_{\mathbf{c} \in \mathbf{C}} y_{\mathbf{c}}=\mathbf{N}_{j}$ & $\forall \mathbf{C}$ & ACI constraint & $(13)$ \\
\hline
\end{tabular}

Model 1: A constraint satisfaction model for determining constructibility of an ACI vector. The model determines whether site occupancies can be selected such that the constraints are not violated.

In a more complex CE model we can test the constructibility of an ACI vector with a constraint satisfaction model, as shown in Model 1. The binary site variables (7) are constrained to the desired stoichiometry (10) by Equation (11). The cluster activity variables (8) are related to the site variables by a logical AND constraint (12), which is equivalent to the relation $y_{\mathbf{c}}=\prod_{i \in \mathbf{c}} x_{i}$. Lastly, the site activity constraints are related to the ACI vector (9) by Equation (12). The model is satisfiable if and only if the ACI is constructible. By using standard translations [36, 37] for the logical AND constraint in Equation (13), the model can be formulated either as a pseudo-Boolean satisfiability problem [37] or a Mixed Integer Programming [38, 39] (MIP) problem. For this application the methods are equivalent, and both can quickly determine the satisfiability of the model. In this work we have used the Gurobi 7.0 MIP solver [40].

\begin{tabular}{|r|l|}
\hline Input: & Site geometry, clusters, desired stoichiometry. \\
\hline 1 & Identify linearly independent set of clusters \\
\hline 2 & Enumerate all ACI vectors \\
\hline
\end{tabular}




\begin{tabular}{|r|l|}
\hline 3 & Determine constructibility of remaining ACI vectors using Model 1. \\
\hline 4 & Calculate convex hull of constructible ACI vectors \\
\hline Return: & Vertices of convex hull (minimizing configurations) \\
\hline
\end{tabular}

Algorithm 1: for finding all minimizing configurations of a CE model.

The method for determining all minimizing configurations is summarized in Algorithm 1. It is intended as a general recipe rather than a strict set of guidelines. Indeed, a variety of application specific optimizations can be applied at each step. For example, if an ACI can be decomposed into subsets, it may be advantageous to test constructibility (step 3) of the subsets separately. Standard convex hull algorithms may be used to maintain a boundary set of ACI vectors, which may permit entire regions of non-boundary ACI vectors to be discarded. Since these optimizations are inherently application specific, we do not discuss them in detail. Rather, we describe some chosen optimizations for the applications presented in the next sections.

This approach provably finds all possible ground states given a choice of interatomic potential class. In addition, the approach is flexible to consider complex interatomic potentials and can be implemented using commercial solvers for constraint satisfaction problems.

\section{Application to a Hexagonal Lattice}

First, we demonstrate the approach in a very simple model system which is still non-trivial: determining the minimizing configurations in a 2D hexagonal lattice model with nearest-neighbor and next-nearest-neighbor pairwise bonds. We fix the number of solute atoms to 24 on a $12 x 12$ lattice. The ACI vector contains two linearly independent parameters, $k_{1}$ and $k_{2}$, which denote the number of active clusters (the number of B-B bonds) of the nearest-neighbor and next-nearest-neighbor types respectively. Rather than test all possible ACI vectors for constructibility, a MIP model is used to identify the maximum and minimum values of $k_{1}$ for every value of $k_{2}$. By doing so, we limit the number of constructibility tests to a small region of ACI space.

Figure 2: All constructible ACI vectors (marked with a blue dot) in a $12 \times 12$ hexagonal lattice with nearest-neighbor and next-nearest-neighbor bonds and a fixed concentration of 24 solute atoms. The ACI vector $\mathbf{N}=k_{1}, k_{2}$ specifies the number of active clusters of each type. The convex hull is marked by a blue line; due to the maximum principle, each vertex of the convex hull represents a minimizing configuration.

Figure 3: Design map for the system. The sectors show the regions of ECI space for which each minimizing configuration is a ground state. All ECI vectors $\mathbf{E}=E_{1}, E_{2}$ are shown in the range $0 \leq|E| \leq 1$. Since the design map is a circular function, a single energetic parameter would suffice to describe any configuration.

Figure 4: All minimizing configurations for a $12 \times 12$ 2D hexagonal lattice with 24 solute atoms. Each configuration lies at a vertex of the convex hull, shown in Figure 2. ${ }^{\dagger}$ Bulk precipitation configurations form a continuum in the continuum limit; there are only two states here, due to the small number of solute atoms.

Figure 2 shows the space of constructible ACI vectors for the chosen lattice model. The ECIs determine an optimal direction in ACI space. A configuration which is extreme in the optimal direction is the ground state for the chosen ECIs. Here, the convex hull of the constructible vectors clearly identifies six minimizing configurations, which are the vertices of the convex hull. The minimizing configurations are also shown in energy space in Figure 3. For a specified set of ECIs, the ground state configuration can be determined from this figure, which essentially constitutes a design map. In the general case, the boundary between two minimizing configurations, $\left\langle\mathbf{E}, \mathbf{N}_{2}-\mathbf{N}_{1}\right\rangle=0$ (a hyperplane of equal energy), intersects the origin. Any design map is therefore a hyperspherical 
function, and all ECI vectors can be normalized without loss of generality. Thus, for a system with $m$ linearly independent clusters, $m-1$ energetic parameters are sufficient to describe any configuration.

Figure 4 shows the solute configurations of the six minimizing configurations. The 'disordered mixing' configuration has a preference for heteroatomic bonding at both neighbor distances. A preference for heteroatomic nearest-neighbor bonds and homoatomic next-nearest-neighbor bonds produces the 'ordered mixing' configuration. The two bulk precipitation configurations exhibit preferences for homoatomic bonding at both neighbor distances, but in varying degrees; in the thermodynamic continuum limit, these configurations would also form a continuum of states. The 'lines' configuration requires a careful balance of bond preferences and, furthermore, exploits the periodic boundary conditions of the lattice to achieve an extreme ACI vector. Lastly, the 'triplets' configuration has a preference for homoatomic bonding at the nearest-neighbor distance only. These six states represent all possible ground state configurations in a nearest-neighbor and next-nearest neighbor pairwise bonding model on a 2D hexagonal lattice.

\section{Application to a Bicrystal in a Hexagonal Lattice}

Figure 5: Hexagonal bicrystal lattice studied for finding complexion types in 2D. The grain boundary, which lies along the [10] line, has been highlighted with a dashed line and by widening the gap between the lines of atoms. Periodic boundary conditions have been applied along the principal axes.

In addition to provably finding all minimizing configurations in a standard Ising model, this approach can be applied to more complex lattice problems, such as those containing interfaces where the cluster expansion is not consistent throughout the lattice. We consider the minimizing configurations at a grain boundary, a problem that we have studied previously using Monte Carlo simulations [41, 42 43, 44]. In this lattice model, a hexagonal lattice with a single grain boundary is considered, as shown in Figure 5. The grain boundary in this 2D lattice is a line which bisects the lattice where any bonds between atoms on either side of this line are defined as grain boundary bonds (otherwise they are defined as intracrystalline). The model has a total of six different bond types; between each nearestneighbor pairing of solvent and solute atoms (A and B) of either crystalline (c) or grain boundary (gb) type. At a fixed solute concentration, this bonding model has three linearly independent clusters; we can specify the ACI vector by $\mathbf{N}=n_{\mathrm{gb}}, k_{\mathrm{gb}}, k_{\mathrm{c}}$, where $n_{\mathrm{gb}}$ is the number of grain boundary sites occupied by solute atoms, and $k_{\mathrm{gb}}$ and $k_{\mathrm{c}}$ are the number of nearest-neighbor $\mathrm{B}-\mathrm{B}$ bonds of the grain boundary and intracrystalline types respectively.

Our goal is to find all minimizing states of the $12 \times 12$ hexagonal lattice with 28 solute atoms (i.e. a concentration of $\approx 20 \%$ solute) under this nearest-neighbor bicrystal potential. This solute concentration is sufficiently large that the grain boundary can be entirely filled with solute atoms. At this concentration, the number of possible configurations is of the order $10^{30}$. Thus, constructing each configuration directly is intractable, which motivates the use of the method described in the previous section. On the other hand, if we consider just the ACI vectors, the number of possible configurations is substantially reduced to 12337 possible ACI vectors for $n_{\mathrm{b}}=28$, which is a small enough set to test for constructibility.

Figure 6: All minimizing ACI vectors in a $12 \times 12$ hexagonal bicrystal lattice with nearestneighbor bonds and a fixed concentration of 28 solute atoms. The ACI vector $\mathbf{N}=n_{\mathrm{gb}}, k_{\mathrm{gb}}, k_{\mathrm{c}}$ specifies the number of grain boundary sites occupied by solute atoms, and the number of B-B bonds of the grain boundary and intracrystalline types. Each vertex of the convex hull represents a minimizing configuration.

Figure 7: All minimizing configurations for a $12 \times 12$ 2D hexagonal lattice with 28 solute atoms, with complexions with a preference for A-B grain boundary bonds and B-B grain boundary bonds placed in separate rows. Solvent and solute atoms are colored gray and blue respectively. ${ }^{\dagger}$ Wetting 
complexions form a continuum as the precipitate can intersect the grain boundary in several ways; as such, we have not given them different names.

Using Model 1, we find that there are 7085 constructible ACI vectors. Note the very large increase in the complexity of the configuration space as compared with the interface-free hexagonal lattice; that system had only 2133 ACI vectors expressable in a 2D space, whereas the addition of the grain boundary requires approximately 3 times as many ACI vectors, which lie in a 3D space. The convex hull of these constructible ACI vectors contains exactly 12 vertices, as shown in Figure 6 . These are the minimizing configurations of the CE model, shown in Figure 7. In a single-crystal model with nearest-neighbor pairwise bonds, only two states are formed: solute precipitation and solid solution. These states are also found here in the bicrystal model (bulk precipitation and bulk mixing); the addition of the grain boundary, even with only nearest neighbor interactions, introduces several new configurations that are ground states for some combination of energies for nearest neighbor bonds. Of these ground states, in particular several ordered states at the grain boundary, often called complexions [21], are found. The energies of the 6 bond types determine which ordered state forms, and in solving the inverse problem, a full mapping between the bond energy space and ground state configuration space is developed and can be read directly from the convex hull.

The 'dots' complexion type is characterized by a preference for A-B grain boundary bonds and a large penalty for B-B crystalline bonds, which leads to solute being separated along the grain boundary. An increase in the preference for A-B grain boundary bonds can bring more atoms to the grain boundary, producing a 'repulsive line' complexion. In this complexion, the preference for A-B grain boundary bonds is greater than the aversion to B-B crystalline bonds, meaning that B-B crystalline bonds will form, though only at the grain boundary. Changing the aversion to B-B crystalline bonds to a preference will produce an 'attractive line' complexion. Here, the preference for A-B grain boundary bonds is significantly stronger than the preference for B-B crystalline bonds. Reducing the preference for A-B grain boundary bonds results in a surface wetting complexion. In a $12 \times 12$ hexagonal lattice, only one surface wetting complexion exists, though in a larger lattice with more solute atoms the number of wetting complexions is larger, as the other two energetic parameters can change the shape of the precipitate. The surface wetting complexion shown here has the same number of B-B crystalline bonds as the bulk precipitation state, but with a weak preference for A-B grain boundary bonds that leads to its formation at the grain boundary.

The counterpart to 'dots' when B-B grain boundary bonds are preferred is the 'dumbbells' complexion, characterized by a preference for B-B grain boundary bonds and a large penalty for B-B crystalline bonds. Successive reductions in the penalty for B-B crystalline bonds again allow for greater grain boundary segregation, which results in the 'armchairs' and 'repulsive lines' complexion types respectively. Changing the penalty for B-B crystalline bonds to a preference produces an 'attractive lines' complexion type. Increasing the strength of this preference results in interior wetting. Trends in proclivities for particular types of bonds shown in Figure 7 will be studied in more detail in future work.

Solving for ground states using the approach outlined in Algorithm 1 was well-suited to this problem. It is possible that we could have found all the minimizing configurations by sampling the bond energy space very finely, and finding the ground state configuration at each sample, though we would not have been able to guarantee that other ground states do not exist between samples. The approach presented here provably finds all minimizing configurations and the catalog in Figure 7 is therefore complete. Alternatively, the polytope method could have been used to determine all possible ground states, but defining the compatibility between clusters at the grain boundary and clusters in the crystalline regions of the lattice would have been non-trivial. Model 1 allows for a non-homogeneous potential as in the case of the bicrystal lattice, and moreover by casting this problem in terms of constraint satisfaction, a MIP model could be used to find all the constructible states.

The application presented here is a small CE model with effectively 2 clusters. However, enumeration of minimizing configurations is only of interest if the number of clusters is small, since this leads leads to a small number of minimizing configurations which can provide physical insight into the system being modelled. If the CE model contains many clusters, the number of minimizing 
configurations is large and most of them are not physically realizable and do not warrant consideration. Potential applications of this model include solving for all possible ground states in multicomponent alloys, compounds, and nanoparticles.

\section{Conclusions}

We have introduced a method for rigorously solving the inverse lattice problem to find all possible ground states given a specific class of cluster expansion. First we formalized the inverse problem for the cluster expansion model and showed that the inverse problem can be solved by determining all possible energetically distinct configurations, where all possible ground states lie on the convex hull in configuration space. To determine all distinct configurations, we considered the problem in cluster space, where counts of each type of cluster (termed an active cluster instance, ACI) are used to consider only configurations of different energy. The lattice model problem is cast into a constraint satisfaction problem to determine if it is possible to construct a configuration with a given set of ACI counts. Once configuration space is reduced to only constructible, energetically distinct configurations, the convex hull can be constructed and all possible ground states can be determined. To demonstrate this method, we determined all possible ground states in a bicrystal lattice model with nearest-neighbor interactions. In doing so, we showed how this method provably determines all possible ground states even in a non-homogeneous cluster expansion lattice problem. Software demonstrating the use of the method is available online [45].

\section{Acknowledgements}

This work was supported by the U.S. Army Research Office under grant W911NF-14-1-0539. P.M.L. received funding for an international research stay from the Otto Mønsteds Fond foundation. A.R.K received funding from a National Defense Science and Engineering Fellowship.

\section{References}

[1] E. Ising, Beitrag zur theorie des ferromagnetismus, Z. Phys. A 31 (1) (1925) 253-258.

[2] J. Sanchez, F. Ducastelle, D. Gratias, Generalized cluster description of multicomponent systems, Physica A 128 (1-2) (1984) 334-350. doi : \{10.1016/0378-4371(84) 90096-7\}.

[3] G. Ceder, A derivation of the ising model for the computation of phase diagrams, Comput. Mater. Sci. 1 (2) (1993) 144-150.

[4] J. Connolly, A. Williams, Density-functional theory applied to phase-transformations in transitionmetal alloys, Phys. Rev. B 27 (8) (1983) 5169-5172. doi : \{10 .1103/PhysRevB . 27. 5169$\}.$

[5] K. Terakura, T. Oguchi, T. Mohri, K. Watanabe, Electronic theory of the alloy phase-stability of cu-ag, cu-au, and ag-au systems, Phys. Rev. B 35 (5) (1987) 2169-2173.

doi: $\{10.1103 /$ PhysRevB.35.2169\}.

[6] S.-H. Wei, L. Ferreira, A. Zunger, First-principles calculation of temperature-composition phase diagrams of semiconductor alloys, Phys. Rev. B 41 (12) (1990) 8240.

[7] M. Asta, C. Wolverton, D. De Fontaine, H. Dreyssé, Effective cluster interactions from clustervariation formalism. i, Phys. Rev. B 44 (10) (1991) 4907.

[8] M. Asta, R. McCormack, D. de Fontaine, Theoretical study of alloy phase stability in the cd-mg system, Phys. Rev. B 48 (2) (1993) 748. 
[9] V. Ozoliņ̌s, C. Wolverton, A. Zunger, Cu-au, ag-au, cu-ag, and ni-au intermetallics: Firstprinciples study of temperature-composition phase diagrams and structures, Phys. Rev. B 57 (11) (1998) 6427.

[10] M. Asta, J. Hoyt, Thermodynamic properties of coherent interfaces in fcc-based ag-al alloys: a first-principles study, Acta materialia 48 (5) (2000) 1089-1096.

[11] A. van de Walle, G. Ceder, Automating first-principles phase diagram calculations, J. Phase Equilib. 23 (4) (2002) 348.

[12] G. Ghosh, A. Van de Walle, M. Asta, First-principles calculations of the structural and thermodynamic properties of bcc, fcc and hcp solid solutions in the al-tm (tm= ti, zr and hf) systems: a comparison of cluster expansion and supercell methods, Acta Materialia 56 (13) (2008) 3202-3221.

[13] B. P. Burton, A. van de Walle, First principles phase diagram calculations for the octahedralinterstitial system $\alpha$ tio x, 0 x 1/2, Calphad 39 (2012) 97-103.

[14] S. Allen, J. Cahn, Ground state structures in ordered binary alloys with second neighbor interactions, Acta Metall. 20 (3) (1972) 423-433.

[15] F. Ducastelle, Order and phase stability in alloys, in: Interatomic Potential and Structural Stability, Springer, 1993, pp. 133-142.

[16] M. Kaburagi, J. Kanamori, A method of determining the ground state of the extended-range classical lattice gas model, Prog. Theor. Phys. 54 (1) (1975) 30-44.

[17] H. Ackermann, G. Inden, R. Kikuchi, Tetrahedron approximation of the cluster variation method for bcc alloys, Acta Metallurgica 37 (1) (1989) 1-7.

[18] G. Ceder, G. D. Garbulsky, D. Avis, K. Fukuda, Ground states of a ternary fcc lattice model with nearest-and next-nearest-neighbor interactions, Phys. Rev. B 49 (1) (1994) 1.

[19] G. Zhang, F. Stillinger, S. Torquato, The perfect glass paradigm: Disordered hyperuniform glasses down to absolute zero, Scientific Reports 6.

[20] M. Hejna, P. J. Steinhardt, S. Torquato, Nearly hyperuniform network models of amorphous silicon, Physical Review B 87 (24) (2013) 245204.

[21] P. R. Cantwell, M. Tang, S. J. Dillon, J. Luo, G. S. Rohrer, M. P. Harmer, Grain boundary complexions, Acta Mater. 62 (2014) 1-48.

[22] W. D. Kaplan, D. Chatain, P. Wynblatt, W. C. Carter, A review of wetting versus adsorption, complexions, and related phenomena: the rosetta stone of wetting, Journal of Materials Science 48 (17) (2013) 5681-5717. 
[23] T. Frolov, M. Asta, Y. Mishin, Segregation-induced phase transformations in grain boundaries, Phys. Rev. B 92 (2) (2015) 020103.

[24] Z. Pan, T. J. Rupert, Effect of grain boundary character on segregation-induced structural transitions, Phys. Rev. B 93 (13) (2016) 134113.

[25] Z. Pan, T. J. Rupert, Formation of ordered and disordered interfacial films in immiscible metal alloys, Scripta Materialia 130 (2017) 91-95.

[26] Z. Pan, T. J. Rupert, Amorphous intergranular films as toughening structural features, Acta Materialia 89 (2015) 205-214.

[27] N. Zhou, Z. Yu, Y. Zhang, M. P. Harmer, J. Luo, Calculation and validation of a grain boundary complexion diagram for bi-doped ni, Scripta Materialia 130 (2017) 165-169.

[28] A. Kundu, K. M. Asl, J. Luo, M. P. Harmer, Identification of a bilayer grain boundary complexion in bi-doped cu, Scripta Materialia 68 (2) (2013) 146-149.

[29] J. Rickman, H. Chan, M. Harmer, J. Luo, Grain-boundary layering transitions in a model bicrystal, Surface Science 618 (2013) 88-93.

[30] P. R. Cantwell, S. Ma, S. A. Bojarski, G. S. Rohrer, M. P. Harmer, Expanding time-temperaturetransformation (ttt) diagrams to interfaces: A new approach for grain boundary engineering, Acta Materialia 106 (2016) 78-86.

[31] W. Huang, D. A. Kitchaev, S. T. Dacek, Z. Rong, A. Urban, S. Cao, C. Luo, G. Ceder, Finding and proving the exact ground state of a generalized Ising model by convex optimization and MAXSAT, Phys. Rev. B 94 (13). doi : \{10.1103/PhysRevB. 94.134424$\}.$

[32] E. Boros, P. Hammer, Pseudo-boolean optimization, Discret. Appl. Math. 123 (1-3) (2002) 155225. doi: $\{10.1016 / \mathrm{s} 0166-218 \mathrm{X}(01) 00336-5\}$.

[33] T.-D. Lee, C.-N. Yang, Statistical theory of equations of state and phase transitions. II. Lattice gas and Ising model, Physical Review 87 (3) (1952) 410.

[34] B. Grünbaum, V. Klee, M. A. Perles, G. C. Shephard, Convex polytopes, Vol. 16, Springer, 1967.

[35] R. T. Rockafellar, Convex Analysis, Princeton University Press, 1970.

[36] G. G. Brown, R. F. Dell, Formulating integer linear programs: A rogues' gallery, INFORMS Trans. Educ. 7 (2) (2007) 153-159.

[37] J. Marques-Silva, Practical applications of boolean satisfiability, in: Discrete Event Systems, 2008. WODES 2008. 9th International Workshop on, IEEE, 2008, pp. 74-80. 
[38] G. L. Nemhauser, L. A. Wolsey, Integer Programming and Combinatorial Optimization, John Wiley \& Sons, 1988.

[39] D.-S. Chen, R. G. Batson, Y. Dang, Applied integer programming: modeling and solution, John Wiley \& Sons, 2010.

[40] Gurobi Optimization, Inc., Gurobi optimizer reference manual (2016).

URL http: //www.gurobi.com

[41] T. Chookajorn, C. A. Schuh, Thermodynamics of stable nanocrystalline alloys: A Monte Carlo analysis, Phys. Rev. B 89 (6). doi: $\{10.1103$ / PhysRevB. 89.064102$\}$.

[42] A. R. Kalidindi, C. A. Schuh, A compound unit method for incorporating ordered compounds into lattice models of alloys, Comput. Mater. Sci. 118 (2016) 172-179.

[43] A. R. Kalidindi, C. A. Schuh, Stability criteria for nanocrystalline alloys, Acta Mater. 132 (2017) $128-137$.

[44] A. R. Kalidindi, C. A. Schuh, Phase transitions in stable nanocrystalline alloys, J. Mater. Res. (2017) 1-10.

[45] P. M. Larsen, A. R. Kalidindi, S. Schmidt, C. A. Schuh, Example code repository (2017).

URL http://github.com/pmla/alloy-design 